\title{
ASTROPHYSICAL APPLICATIONS OF POWERFUL NEW ATOMIC DATABASES
}

Chairperson and Editor: W.L. WIESE 
JD 16

\title{
ASTROPHYSICAL APPLICATIONS OF POWERFUL NEW ATOMIC DATABASES
}

\author{
Prepared by W.L. Wiese
}

Scientific Organizing Committee: S.J. Adelman (co-Chairperson, USA), B. Baschek (Germany), P. Demarque (USA), D. Lambert (USA), J. Percy (Canada)\& W.L. Wiese (coChairperson, USA)

Supporting Commissions: 14 (Atomic \& molecular data), 27 (Variable stars), 29 (Stellar spectra), 35 (Stellar constitution) \& 36 (Theory of stellar atmospheres)

Several comprehensive atomic and molecular databases have been recently completed which contain radiation data as well as other atomic data for millions of spectral lines, such as the International Opacity Project and the Smithsonian Database. Also, several smaller but still substantial sets of data have been produced, and most of these are of greatly improved accuracy and thus enable astronomers to pursue new problems or solve existing ones at a much greater level of confidence level and in much more detail. Many of these data are now being made available both in printed tabulations and in electronic form. Some applications to astrophysical problems have already taken place. It is thus interesting and timely (a) to communicate these new atomic data capabilities to the astronomical community at large, and (b) to report on some initial experiences in utilizing these new data sets on a diverse set of astrophysical topics.

This Joint Discussion has served as a forum to discuss such first experiences gained and at the same time to provide information to astronomers about the scope and quality of these new data, and to highlight further atomic data needs. 21 invited talks were grouped into 3 half-day sessions, and the extended abstracts are given below in the order of the presentations. The invited talks were complemented by 37 poster papers, the titles of which are given at the end. The complete proceedings of this Joint Discussion will be published in 1995 in the Astronomical Society of the Pacific Conference Series. 